\title{
Radiographic features of osteogenesis imperfecta
}

\author{
Armelle Renaud • Julie Aucourt • Jacques Weill • \\ Julien Bigot • Anne Dieux • Louise Devisme • \\ Antoine Moraux • Nathalie Boutry
}

Received: 7 March 2013 /Revised: 24 April 2013 /Accepted: 26 April 2013 /Published online: 19 May 2013

(C) The Author(s) 2013. This article is published with open access at Springerlink.com

\begin{abstract}
Background Osteogenesis imperfecta (OI), commonly called "brittle bone disease", is a genetic disorder characterised by increased bone fragility and decreased bone density due to quantitative and/or qualitative abnormalities of type I collagen. Different types of OI exist, from mild to severe; they may lead to death, multiple bone fractures, skeletal deformity and short stature.
\end{abstract}

\footnotetext{
A. Renaud $\cdot$ J. Bigot $\cdot$ A. Moraux $\cdot$ N. Boutry $(\bowtie)$

Department of Pediatric Radiology, Jeanne de Flandre Hospital, Lille 2 University, University Hospital of Lille, CHRU de Lille, 59037 Lille, France

e-mail: nboutry@gmail.com

\section{N. Boutry}

e-mail: nathalie.boutry@chru-lille.fr
}

\author{
A. Renaud $\cdot$ J. Aucourt $\cdot$ A. Moraux $\cdot$ N. Boutry \\ Department of Musculoskeletal Radiology, \\ Roger Salengro Hospital, Lille 2 University, \\ University Hospital of Lille, Lille, France \\ J. Weill \\ Department of Pediatric Endocrinology, \\ Jeanne de Flandre Hospital, Lille 2 University, \\ University Hospital of Lille, \\ Lille, France
}

\section{A. Dieux}

Department of Genetics, Jeanne de Flandre Hospital,

Lille 2 University, University Hospital of Lille,

Lille, France

L. Devisme

Department of Pathology, Lille 2 University,

University Hospital of Lille,

Lille, France
Methods Severe cases are usually diagnosed before birth and may incite the parents to choose therapeutic abortion, whereas milder cases are much more difficult to diagnose and may be sometimes confused with non-accidental injury (NAI) ("child abuse") in young children. Whatever the degree of severity, conventional radiography still remains the mainstay in diagnosing OI.

Results The prognosis of this disorder has changed in the last few years thanks to biphosphonate therapy.

Conclusion The aim of this pictorial review is to illustrate the radiographic manifestations of OI, including in children receiving biphosphonates, and to outline specific patterns that help differentiate OI from NAI when necessary.

Key Points

- The main radiographic features of OI are osteopenia, bone fractures and bone deformities.

- Some radiographic features depend on the type of OI or may be encountered with biphosphonates.

Keywords Osteogenesis imperfecta · Radiography · Biphosphonates · Child abuse
Abbreviations
OI Osteogenesis imperfecta
NAI Non accidental injury
CT Computed tomography
MRI Magnetic resonance imaging

\section{Introduction}

Also known as "brittle bone disease", osteogenesis imperfecta (OI) is a genetic disorder characterised by increased bone fragility and low bone mass density due to quantitative and/or 
Table 1 Sillence and Glorieux classification of OI

Type II OI (lethal)

Type III OI (severe)

Type IV OI (moderate)

Types V, VII and VII have been added to the original classification system (no type I collagen mutation, but abnormal bone on microscopy and similar phenotype)

Type V OI

Type VI OI

Type VII OI
Fractures; minor deformities

Almost normal stature

Blue sclerae

"Dentinogenesis imperfecta" may be present

Fractures in utero

Death before birth (respiratory deficiency)

Fractures; kyphoscoliosis; major deformities

Very small stature

Triangular face; variable colour of sclerae

"Dentinogenesis imperfecta" is frequent

Fractures

Small stature

Variable colour of sclerae

"Dentinogenesis imperfecta" may be present

Fractures; hyperplastic callus; interosseous membrane ossification; metaphyseal dense lines

Normal colour of sclerae

No "dentinogenesis imperfecta"

Looser striations mimicking fractures

No wormian bones

Fractures; coxa vara

Normal colour of sclerae

No "dentinogenesis imperfecta"

Rhizomelia qualitative abnormalities of type I collagen $[1,2]$. It is usually characterised by an autosomal dominant mode of inheritance (95\% of cases), but some cases are related to autosomal recessive traits or to a spontaneous mutation [3].

OI is rare but far from exceptional, affecting approximately 1 in 10,000-20,000 births. In the vast majority of cases, it results from mutations in either the COL1A1 or the COL1A2 gene, encoding the pro-alpha 1 and pro-alpha 2 chains, respectively. These polypeptide chains form a triple helix of intracellular type I procollagen, which is the precursor of extracellular type I collagen. The latter is a component of many tissues such as bone, dental enamel, eye sclera, skin, tendons and ligaments. Genetic mutations affect the spatial arrangement of the polypeptide chains and consequently alter the biomechanical properties of type I collagen, particularly its resistance to stretching. The
Fig. 1 Sagittal and transverse US scans in a 26-week foetus with femur length measurements below the $3 \mathrm{rd}$ percentile show a shortened and angulated femur with a hypoechoic cleft (arrowhead) suggestive of a fracture (left) and decreased echogenicity of the cranial vault (right). Courtesy of Pr Freddy AVNI, Lille (France)
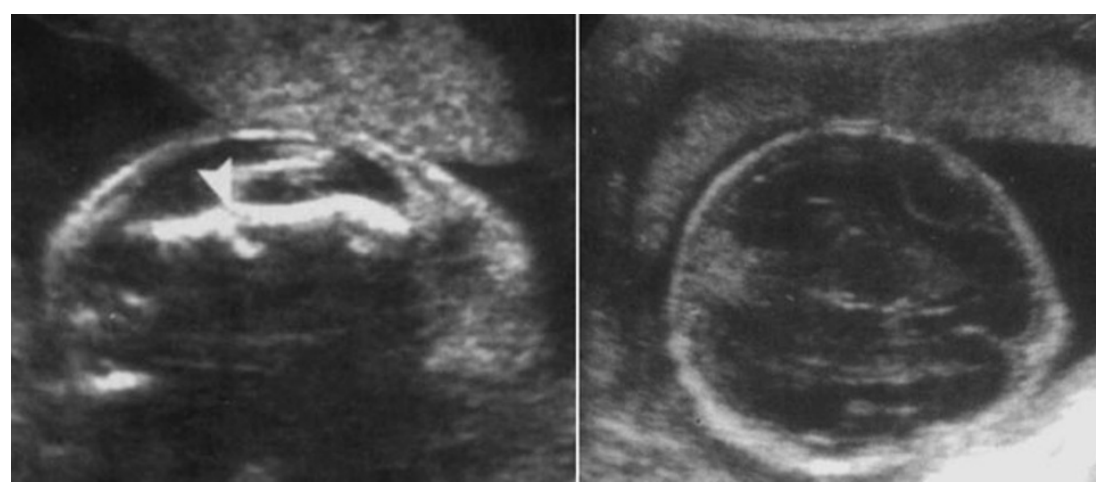

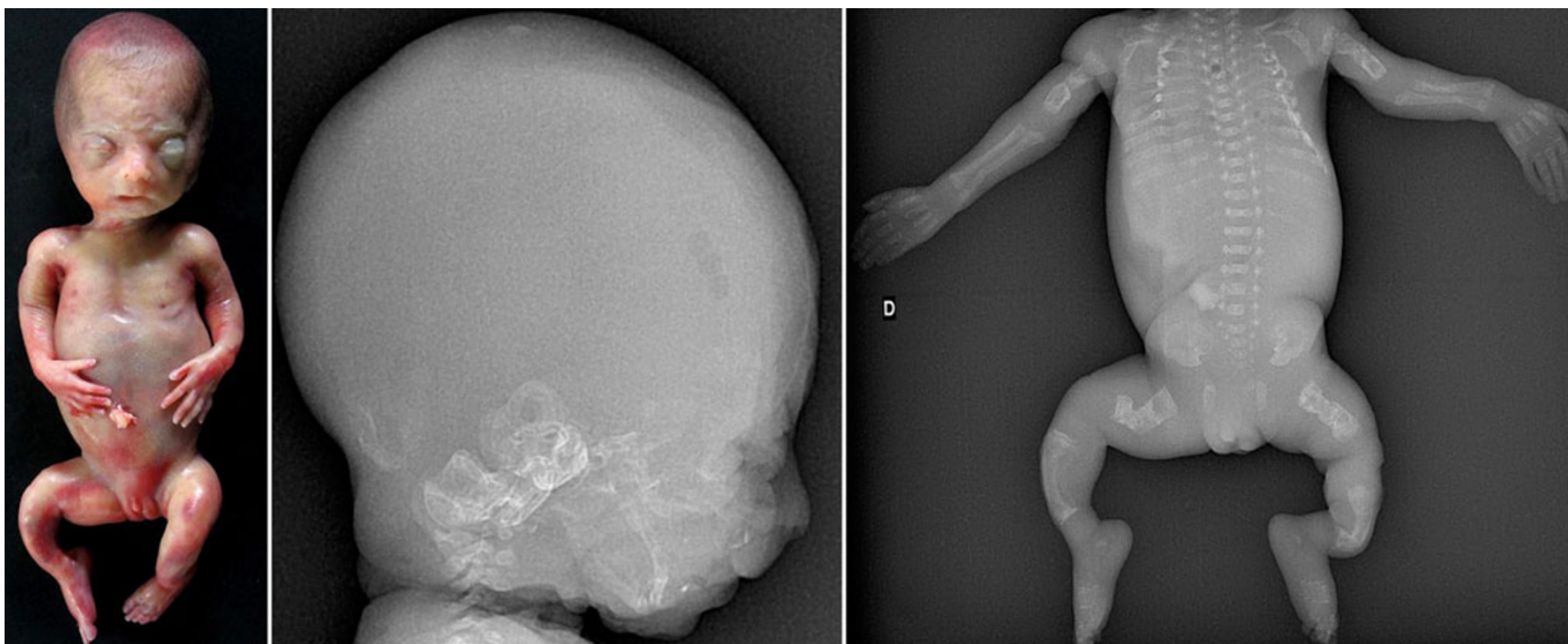

Fig. 2 Postmortem radiographs of a 23-week foetus with lethal OI exhibit a triangular-shaped face and shortened and bowed limbs. On radiographs, there is no mineralisation of the skull vault; severe osteopenia is seen throughout the skeleton with multiple bone fractures and deformities of the ribs and long bones

or at birth (i.e., severe forms of OI) and cases diagnosed after birth (i.e., milder forms of OI).

hallmarks of $\mathrm{OI}$ are therefore bone fragility and other connective-tissue manifestations, with a large variation in phenotype.

The classification most widely used for OI distinguishes four types, based on clinical findings and disease severity (Table 1) [3]. More recently, three types whose phenotype is similar to other types of OI but that are not associated with type I collagen mutations have been added by Glorieux et al. [4] (Table 1). This classification is not always easy to use, as some patients cannot be included (and some genetic mutations are still to be discovered), and it is usually more convenient in practice to distinguish between cases diagnosed before

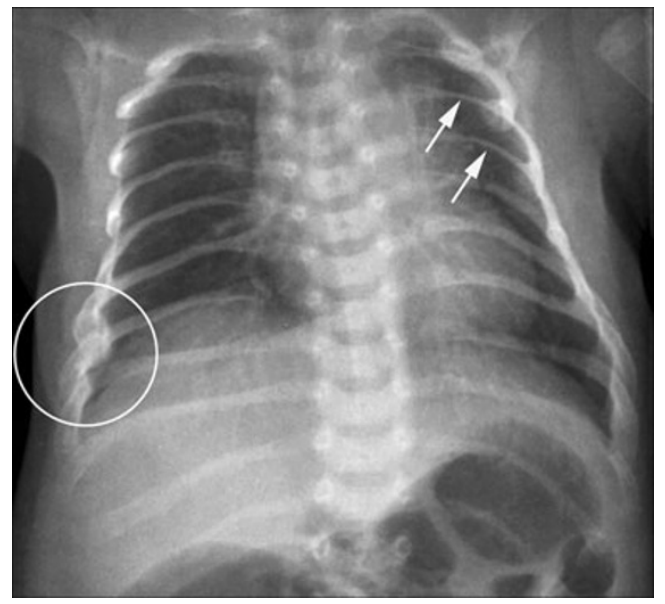

Fig. 3 Anteroposterior radiograph of the chest in a child with OI evidences marked thinning of the posterior ribs (arrows), associated with fractures developing callus

\section{Antenatal diagnosis of OI}

Severe forms of OI (mainly type II) can be diagnosed by ultrasound during the second trimester of pregnancy $[5,6]$. Nonspecific signs such as intra-uterine growth retardation or hydramnios may be seen. Otherwise, examination may show abnormalities of the skull, the rib cage, the spine or the limbs, such as decreased echogenicity due to insufficient mineralisation, deformities related to fractures, callus formation and increased bone plasticity, and micromelia, especially of the femur (Fig. 1) [5, 6]. In case of doubt and when a termination of pregnancy is being considered, low-dose computed tomography (CT) with three-dimensional reconstructions of the whole foetal skeleton can be performed, after 26 weeks of gestation, to yield a correct diagnosis. The role of MRI is limited, except when visualisation of the foetal brain or visceral organs is required to look for associated abnormalities or to assess foetal lung volume [7]. When termination of pregnancy is performed based on the discovery of ultrasound and CT abnormalities, postmortem radiographs are a very useful adjunct to diagnosis, in confirming and specifying foetal bone abnormalities (Fig. 2).

\section{Postnatal diagnosis of $\mathrm{OI}$}

OI encompasses a broad range of presentations, from virtually non-apparent forms to more severe ones, and some may 
Fig. 4 Anteroposterior

radiographs of the pelvis and foot in a child with OI show severe and diffuse osteopenia with prominent thinning of the metatarsal bones

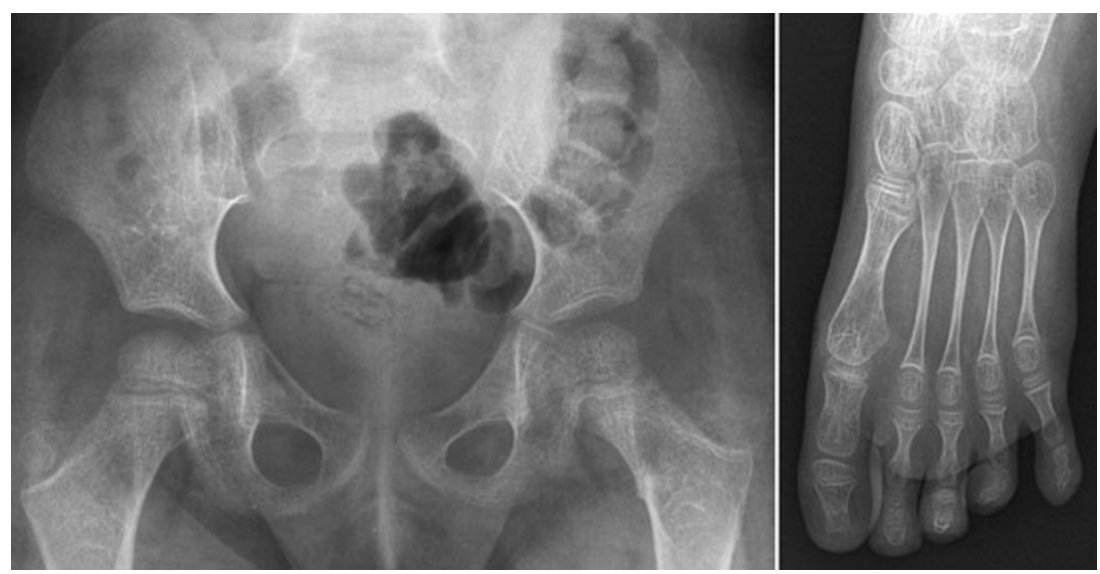

go unnoticed for a long time. Positive diagnosis can be made clinically on the basis of skeletal and/or extraskeletal manifestations, but this is only the case in severe forms of OI. A family history of OI can also be helpful in arriving at a diagnosis. In fact, in most cases, imaging is required for diagnosis.

Extraskeletal manifestations These are inconstant, but their recognition allows a quicker diagnosis of OI. Extraskeletal manifestations are diverse, related as they are to the ubiquitous presence of type I collagen in the human body: blue sclerae (mainly in type I OI); a greyish or yellowish aspect of the teeth, also called "dentinogenesis imperfecta" (mainly in type III OI); skin fragility; joint and ligament hyperlaxity; early hypoacusia;

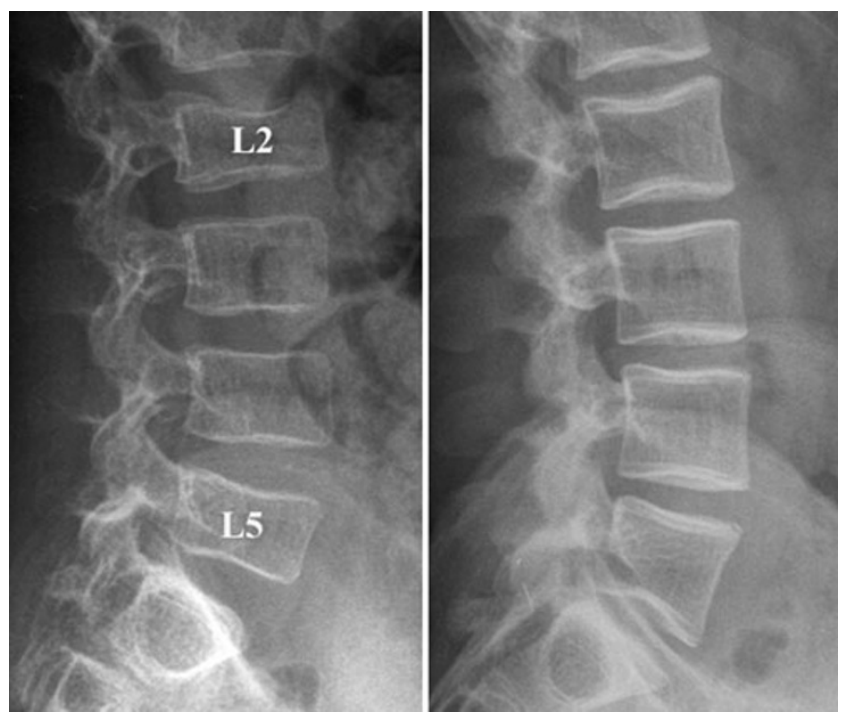

Fig. 5 Lateral radiographs of the spine in two children with OI show homogeneous rarefaction (left) and predominant trabecular rarefaction $(r i g h t)$ of the cortical and trabecular bone, with a "frame-like" pattern of the vertebrae (right). Note the partial collapse of the L2 and L5 vertebral bodies and cardiovascular abnormalities (particularly aortic valve disease) [3, 4, 8]. Less frequently, neurological abnormalities related to basilar impression and platybasia (mainly in type IV OI) or to direct involvement of neurovascular structures may be encountered [9, 10].

Skeletal manifestations OI is generally recognised in children presenting with multiple, repeated or unexplained fractures, especially when the causative trauma is minor or when there is no history of trauma. Other skeletal manifestations depend on the severity of the disease and may include short stature and progressive deformities

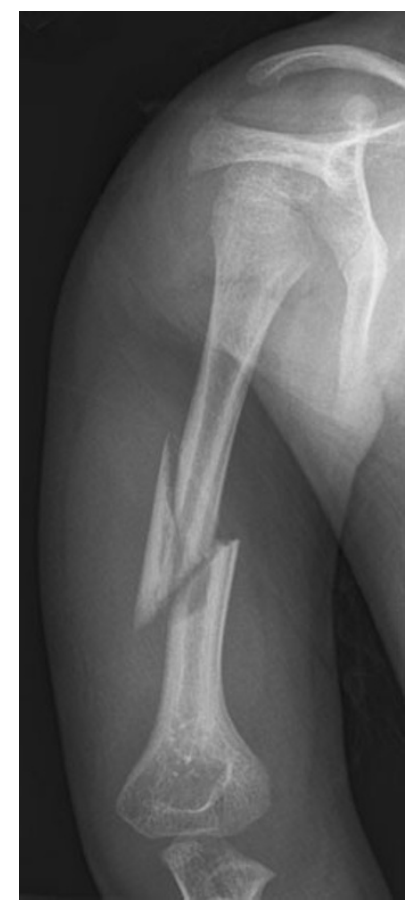

Fig. 6 Anteroposterior radiograph of the humerus in a child with OI reveals a complete fracture of the mid-diaphysis with a detached, triangular fragment 


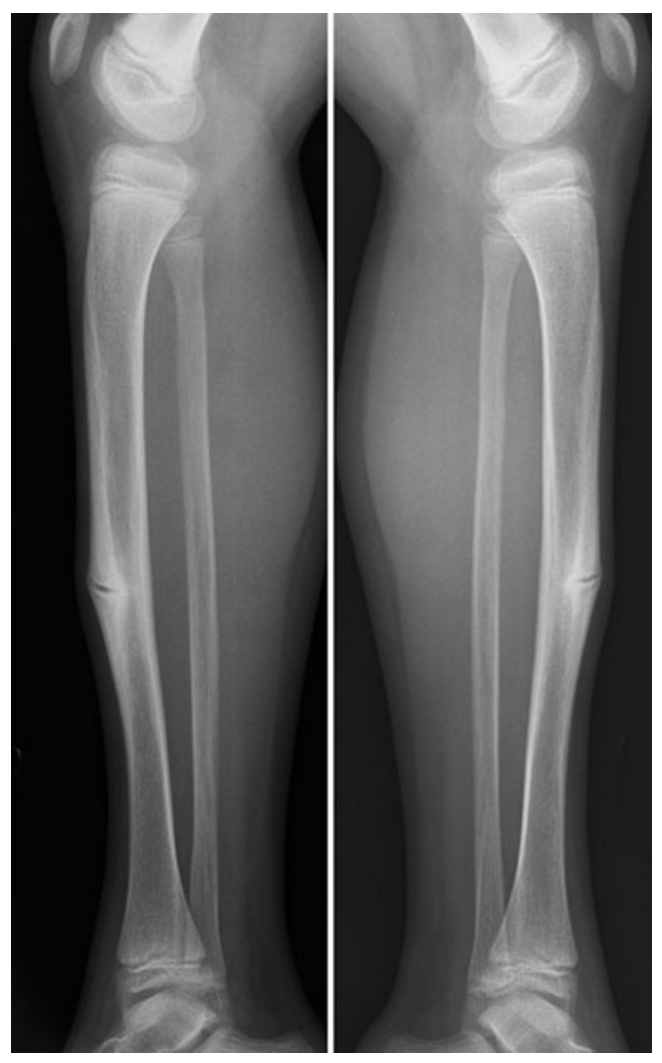

Fig. 7 Lateral radiographs of the leg in a child with OI show a bilateral incomplete fracture of the anterior cortex of the tibial diaphysis

of the spine (kyphosis, scoliosis), the rib cage or the lower limbs (discrepancy in length, bowing).

\section{Radiographic findings in OI}

The main radiographic features are osteopenia, bone fractures and bone deformities. They result from constitutional bone fragility (cortical bone thinning, trabecular bone rarefaction) but also from acquired bone fragility due to muscle wasting and immobilisation. None of them are specific enough, but their association, together with a suggestive clinical history (propensity for fractures, family history of presenile loss of hearing, etc.) may suffice to confirm the diagnosis of OI.

Osteopenia Radiographs reveal cortical bone thinning and excessive trabecular bone transparency (Figs. 3, 4, 5). This finding, however, is subjective and may be difficult to assess with conventional radiography, and its detection requires a significant reduction (about 30 to $50 \%$ ) in calcified bone mass. Bone densitometry by dual-energy $\mathrm{X}$-ray absorptiometry (DEXA) is currently the optimal method to detect decreased bone mineral density, but in

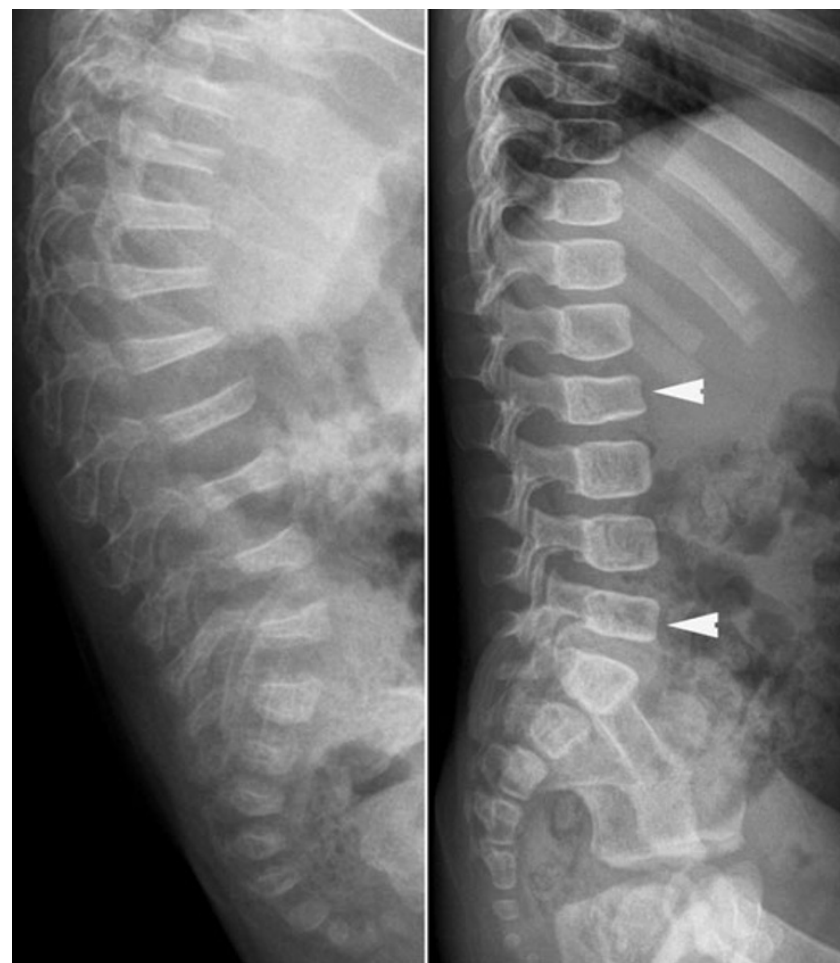

Fig. 8 Lateral radiographs of the spine in two children with OI exhibit severe and multiple vertebral collapses associated with kyphosis (left) and less severe collapses of the vertebral bodies (arrowheads) (right). Osteopenia is much more pronounced on the left

children, accurate interpretation of the results requires a good knowledge of the potential pitfalls related to age, sex, pubertal stage and skeletal maturation [11]. Decreased bone mineral density is not specific for OI; it may be encountered in metabolic disorders (hypogonadism, growth hormone deficiency, hyperthyroidism, juvenile diabetes mellitus, calcium and vitamin D deficiency, etc.). However, when such causes have been excluded, DEXA

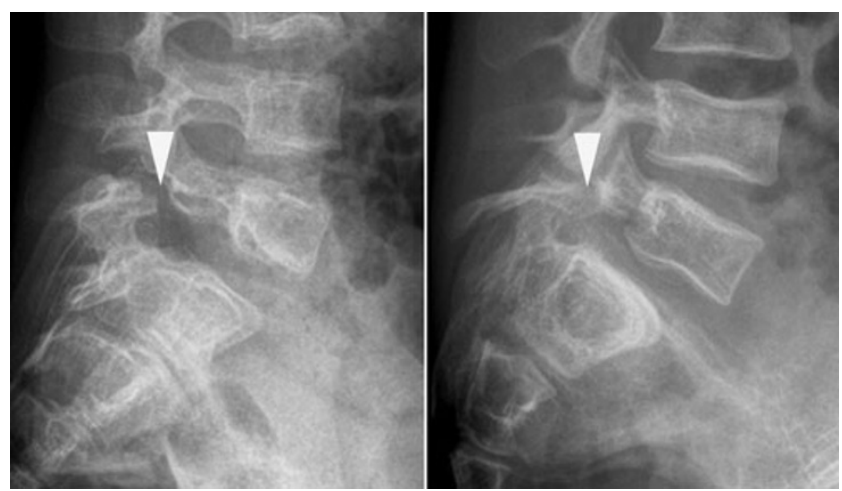

Fig. 9 Lateral radiographs of the lumbosacral junction in two children with OI reveal spondylolysis of L5 (arrowheads) associated with spondylolisthesis 


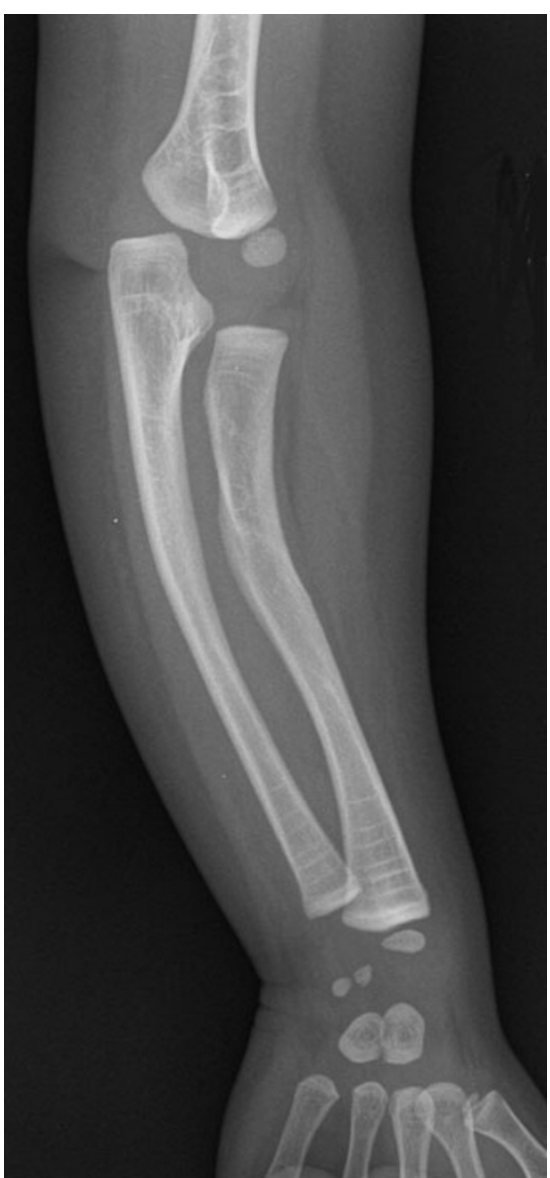

Fig. 10 Anteroposterior radiograph of the forearm in a child with OI exhibits bone deformity and incurvation of the radius and ulna

may help to establish the diagnosis of OI [12] and to monitor the response to biphosphonates.

Bone fractures They affect both the axial and appendicular skeletons. These fractures are similar to those seen in normal children who suffer trauma and usually consolidate within the normally expected times. Some children may have few or no fractures, whereas others experience numerous fractures throughout their lives, especially when they start walking. The most common fractures occur in the long bone diaphyses, the spine and the apophyses. Diaphyseal fractures may be complete (Fig. 6) or incomplete (Fig. 7), and more or less displaced. In the spine, multiple thoracolumbar compression fractures may be seen (Fig. 8). Spondylolysis of L5, with or without consecutive spondylolisthesis, is also common in children with OI due to a fracture (Fig. 9) or an elongation of the pars interarticularis of L5, all of which are fostered by bone fragility and/or hyperlordosis [13, 14]. Apophyseal avulsion fractures are less common; they are often displaced and sometimes bilateral $[15,16]$. They classically involve the olecranon or the tibial tubercle, and usually require internal fixation $[15,16]$.

Bone deformities They most frequently affect the appendicular skeleton, especially the lower limbs, but the upper limbs (Fig. 10) and skull may be involved. These deformities are due to excessive bone malleability and plasticity. In the skull, radiography may show a prominent occipital region (so-called the "Darth Vader" appearance) (Figs. 11, 12) or a flattening of the cranial vault with transverse infolding of the cranial base (the so-called "Tam O'Shanter skull") (Fig. 11); however, such deformities are rare. Much more frequently, radiographs reveal multiple wormian bones (defined as the presence of 10 or more wormian bones [17]) that lend a "mosaic" or "paving" appearance to the cranial vault (Fig. 13). A significant number of wormian bones occur more frequently in more severely affected OI patients [17]. In the long bones, bending (Fig. 14) and thinning of the diaphyses may be seen, sometimes complicated by progressive fractures in the concave aspect of the deformity

Fig. 11 Diagram depicts "Darth Vader" and "Tam O'Shanter" appearances of the cranial vault. The former refers to occipital bone flattening in the sagittal plane (reminiscent of the headgear worn by the film character), whereas the latter refers to cranial vault flattening in the transverse plane with infolding of the skull base (reminiscent of the Scottish bonnet worn by the hero of Burns's poem)

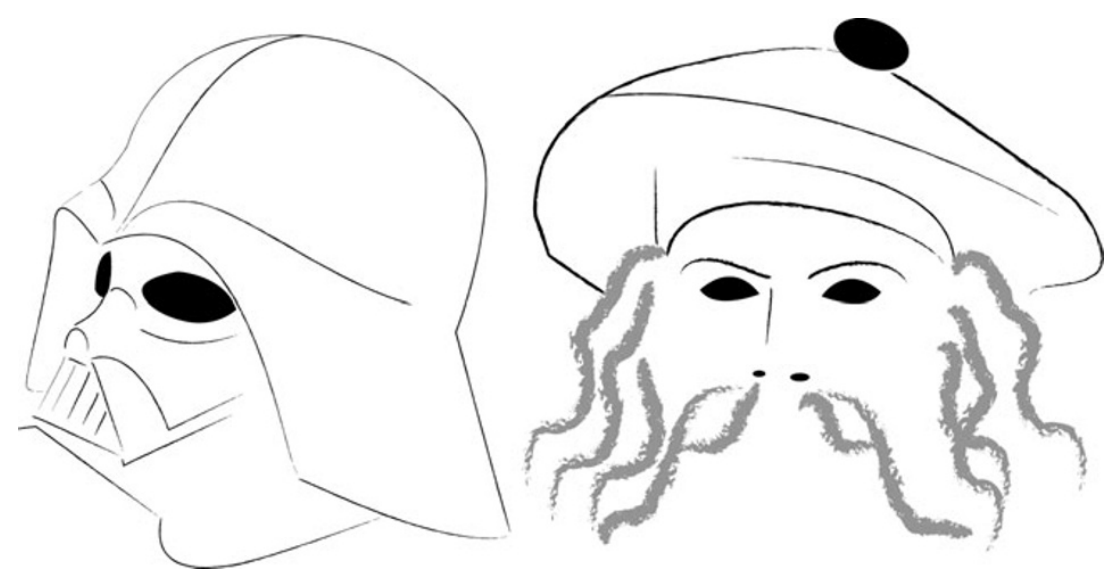




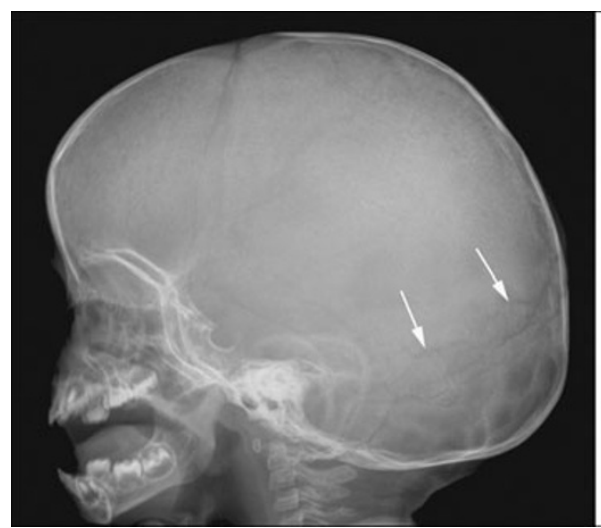

Fig. 12 Lateral radiographs of the skull in a young child with OI (left) and a young adult with OI (right) show an incipient deformation of the occipital region associated with numerous wormian bones (arrows) on the left and a basilar impression on the right, as revealed by significant

that can recur after healing. Severe residual angulation of a healed fracture constitutes another mechanism accounting for long bone deformities. In the lower limbs, diaphyseal bending or angulation may be responsible for lower leg-length discrepancy. In the pelvis, coxa vara and acetabular protrusion have occasionally been reported.

In toddlers with severe forms of OI (mainly type III), the long bones may appear thick and broad, instead of thin and markedly shortened and bowed (Fig. 15). They exhibit a lack of bone modelling with a "bamboo cane appearance" linked to multiple healed fractures and severe bone deformities of the femur (anterolateral bowing or "shepherd's crook" deformity) (Fig. 15) and the tibia (anterior bowing or "saber shin" deformity) (Fig. 16).

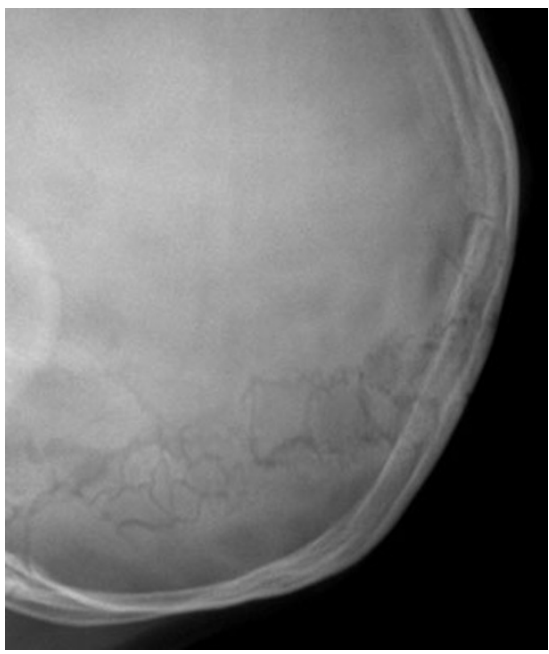

Fig. 13 Lateral radiograph of the skull in a child with OI reveals multiple wormian bones embedded in the lambdoid sutures. This finding is suggestive of the diagnosis, but not specific

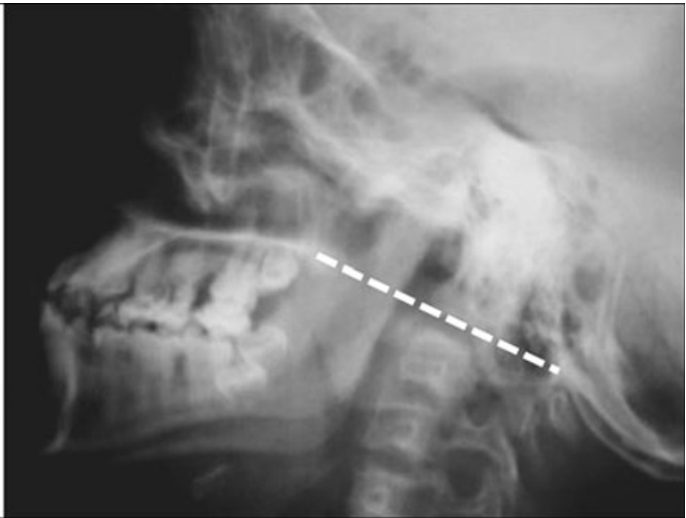

axis migration above Chamberlain's line (i.e., the dotted line joining the posterior aspect of the foramen magnum and the posterior aspect of the hard palate) on the right

\section{Radiographic features depending on the type of OI}

Hyperplastic callus formation This has occasionally been reported in type $\mathrm{V}$ OI, especially in males and in the femur [18-20]. Hyperplastic callus formation can occur either after a fracture (Fig. 17) or surgery or spontaneously, and may mimic osteosarcoma clinically and radiographically. In this case, CT and MRI are useful to

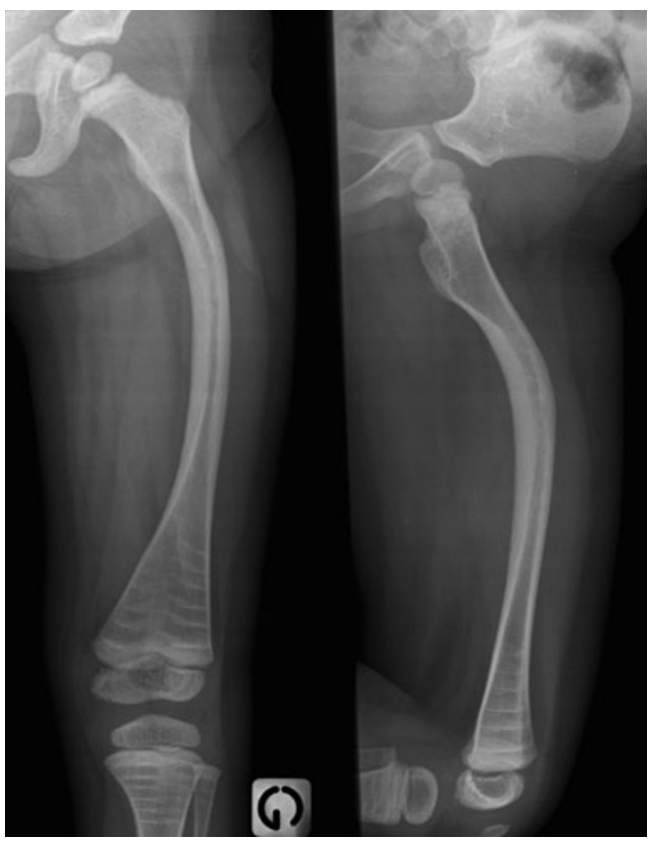

Fig. 14 Anteroposterior and lateral radiographs of the thigh in a child with OI evidence marked deformity of the femur, especially on the lateral view. Note also multiple dense lines in the distal femur and proximal tibia associated with dense metaphyseal bands along the cartilaginous plates related to biphosphonate therapy 


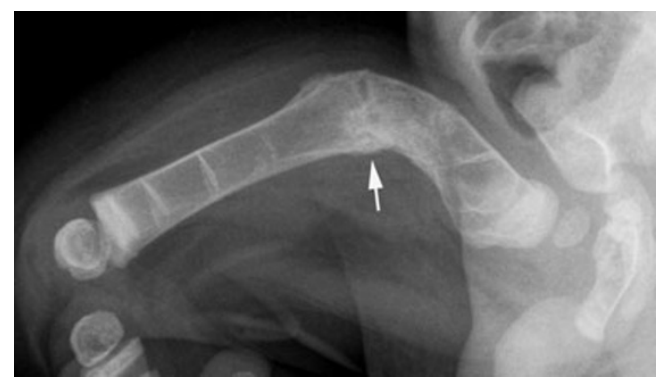

Fig. 15 Lateral radiograph of the thigh in a young child with OI shows considerable deformity of the proximal femur with abnormal bone modelling and an associated fracture developing callus formation (arrow)

avoid misdiagnosis, as they will respectively detect the absence of osteolysis and of bone marrow infiltration. CT may reveal an occult fracture, whereas MRI may show a thick calcified rim with low signal on T1- and T2weighted images at the periphery of the callus [18-20]. Hyperplastic callus formation is not specific to OI; it can also be seen in children with spinal dysraphism or with subperiosteal hematomas related to NAI, bleeding disorders or neurofibromatosis.

Ossifications of the interosseous membrane These are encountered in type V OI, in the forearm (Fig. 18) or leg, and may be associated in some cases with a congenital dislocation of the radial head $[20,21]$.

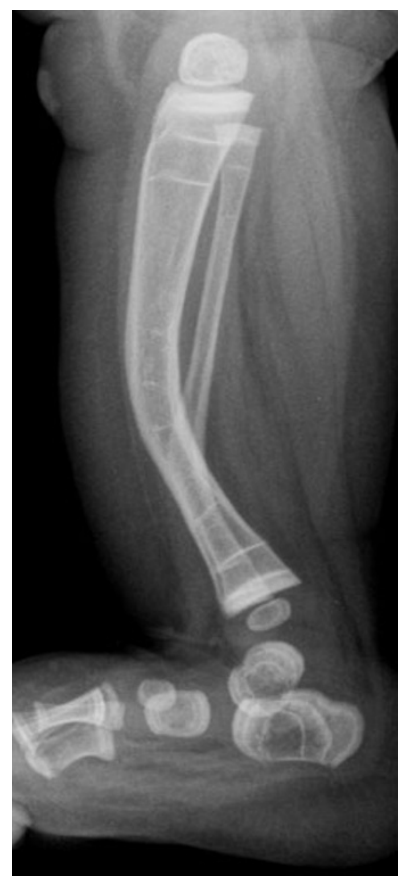

Fig. 16 Lateral radiograph of the leg in a child with OI shows anterior bowing of the tibia
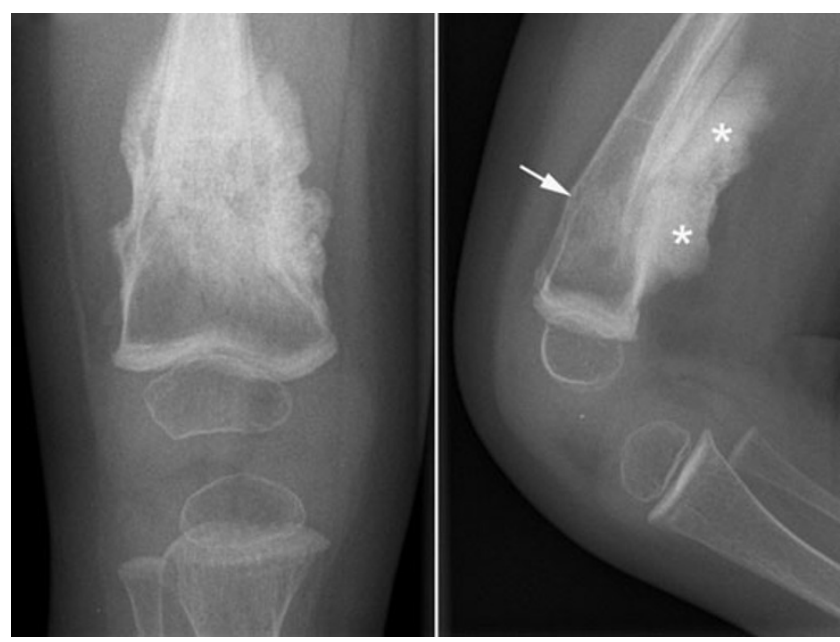

Fig. 17 Anteroposterior and lateral radiographs of the knee in a child with type V OI reveal a hyperplastic callus (asterisks) of the distal femur following a fracture (arrow)

"Popcorn" calcifications They are more commonly seen in type III OI, in the metaphyseal and epiphyseal regions of the knee (Fig. 19), and may contribute to femoral growth deficiency and lower leg-length discrepancy $[22,23]$. These intraosseous calcifications are thought to result from microtraumatic fragmentation and disordered maturation of the growth plate.

Dense metaphyseal bands These are usually encountered in children with OI receiving biphosphonates, but have been reported in type V OI independently of any treatment. Dense metaphyseal bands can also be seen in children receiving biphosphonates for secondary osteoporosis (cerebral palsy, glucocorticoid-treated disorders, etc.).

\section{Radiographic findings with biphosphonate therapy}

Biphosphonates are potent inhibitors of osteoclastmediated bone resorption and have demonstrated clinical usefulness in the treatment of children with severe forms

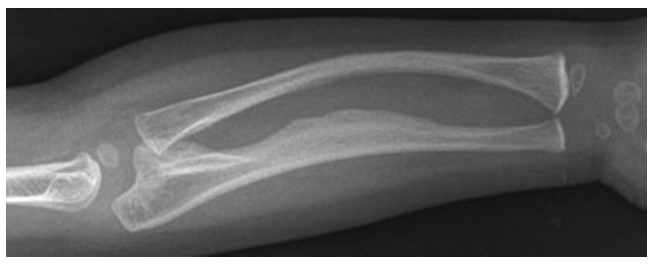

Fig. 18 Anteroposterior radiograph of the forearm in a child with type $\mathrm{V}$ OI shows incipient ossification of the ulnar aspect of the interosseous membrane 


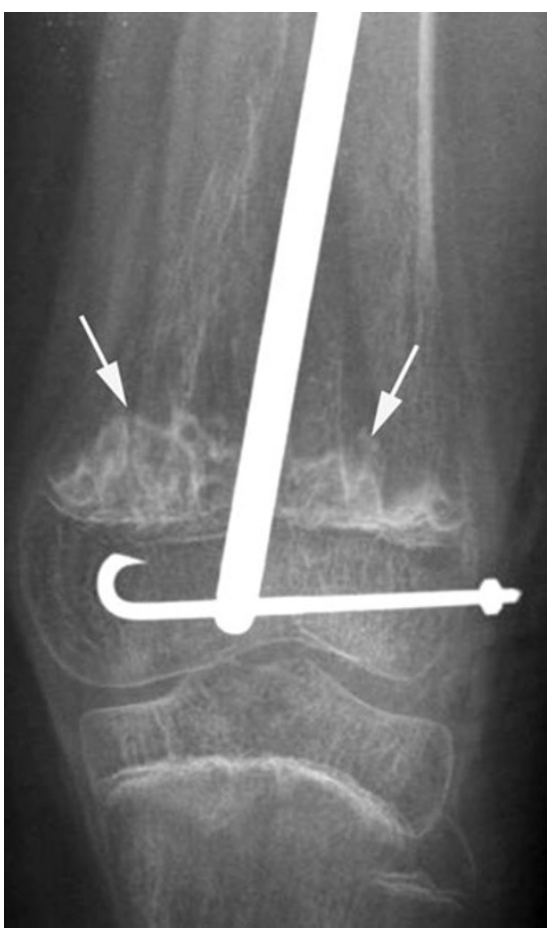

Fig. 19 Anteroposterior radiograph of the knee in a child with type III OI and a history of femoral osteosynthesis evidences "popcorn" calcifications (arrows) with sclerotic margins

of OI [24]. The treatment usually consists of cyclic intravenous infusions and may be initiated after birth. Several studies have reported beneficial effects of biphosphonates on pain, mobility, growth, bone mineral density and bone turnover and fracture rate [25-28]. However, long-term effects of these agents on the immature growing skeleton have to be assessed.

On radiographs, specific findings in children receiving biphosphonates include increased bone density in the spine and long bones, as compared with previous radiographs, dense metaphyseal lines (so-called "zebra lines"), very similar to Harris growth arrest lines, and dense metaphyseal bands. In long bones, these lines are parallel to the growth plate; they are located in the metaphysis and move towards the diaphysis in step with bone growth (Fig. 14). Each dense line corresponds to one intravenous course, and the space between two lines depends on the bone growth rate and delay between two courses. When multiple, these lines are responsible for a "ladder-rung" appearance (Fig. 14). In the spine or in the flat bones, apophyses and epiphyses, a "bone-within-abone" pattern, which may be considered to be the equivalent of metaphyseal dense lines in long bones, may be observed (Fig. 20) [29]. When there is less bone growth between courses of the drug, the metaphyseal lines are more closely spaced and appear as dense metaphyseal bands (Figs. 14, 16) and dense vertebral endplates.

\section{Differential diagnosis of OI}

A positive diagnosis of OI can be difficult, particularly in young children (before the age of 2 years) with mild forms of OI (types I, IV) and when there are few or no obvious extraskeletal manifestations and no familial history of increased bone fragility. These cases of OI may be tragically mistaken for NAI. Indeed, in both OI and NAI, unexplained, multiple and repeated fractures may occur; children may present with bruises and contusions; radiographs may reveal different types of fractures, fractures of various ages that went undetected and no evidence of osteopenia. A thorough radiographic examination, including a skeletal survey, can help differentiate between OI and NAI (Table 2). In NAI, some fractures are very suggestive of abuse (i.e., posterior rib fractures, metaphyseal corner fractures and complex skull fractures) $[30,31]$.
Fig. 20 Lateral radiograph of the spine and oblique lateral radiograph of the foot in a child with OI receiving

biphosphonate therapy reveal a "bone-within-a-bone" pattern of the vertebral bodies and the cuboid bone (arrowheads). Note also the presence of sclerotic lines inside the base of the fifth metatarsal bone (arrow)
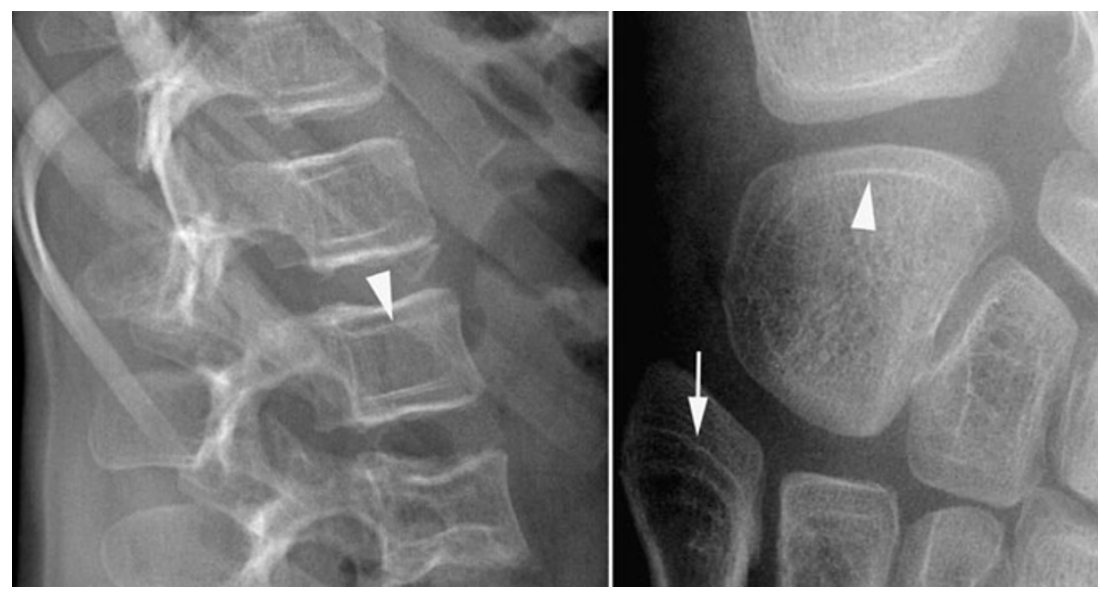
Table 2 Typical and overlapping radiographic findings in OI and NAI

\begin{tabular}{|c|c|c|c|}
\hline Radiographic signs & Typical Ol & Typical NAI & Overlapping \\
\hline Osteopenia & Yes & No & \\
\hline Multiple fractures & Yes & Yes & \\
\hline Fractures of various ages & Yes & Yes & \\
\hline Diaphyseal fractures & Yes & Yes & \\
\hline Classic metaphyseal lesions & No & Yes & \\
\hline Long bone deformities & Yes & No & \\
\hline Skull deformities & Yes & No & \\
\hline Multiple wormian bones & Yes & No & \\
\hline Complex skull fracture & No & Yes & \\
\hline Vertebral fractures & Yes & Yes & \\
\hline Spinous process fractures & No & Yes & \\
\hline Posterior rib fractures & No & Yes & \\
\hline Anterior / lateral rib fractures & Yes & Yes & \\
\hline Hyperplastic callus formation & Yes & Yes & \\
\hline Apophyseal avulsion fractures & Yes & No & \\
\hline Interosseous ossifications & Yes (type V) & No & \\
\hline "Popcorn" calcifications & Yes (type III) & No & \\
\hline Dense metaphyseal bands & Yes (V, BP) & No & \\
\hline
\end{tabular}

$B P$ biphosphonates

Posterior rib fractures Rib fractures usually result from anteroposterior compression of the rib cage when the child is held around the chest and violently shaken back and forth. The posterior involvement is due to excessive leverage of the posteromedial part of the rib over the transverse process of the spine and is highly specific of abuse $[30,31]$. In this situation, posterior rib fractures are frequently multiple on radiographs and are often associated with callus formation when discovered (Fig. 21).
Metaphyseal corner fractures Long bone fractures may be encountered in NAI, but in contrast to OI, where the fractures most commonly affect the diaphyseal regions, they classically involve the metaphysis, especially that of the distal femur (Fig. 22), the proximal and distal tibia and, less frequently, the proximal humerus [30, 31]. Metaphyseal corner fractures, also known as classic metaphyseal lesions, are virtually pathognomonic for abuse [32]. They are due to shearing forces applied to the child's extremities when shaken. The fracture lines 


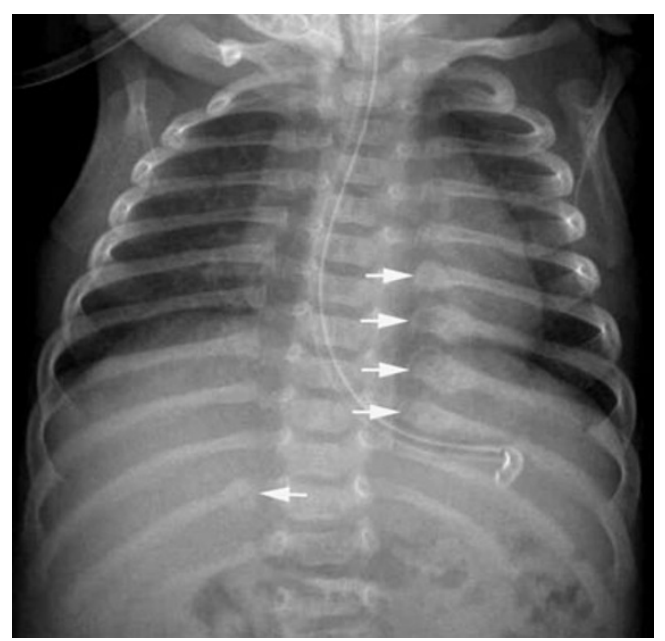

Fig. 21 Anteroposterior radiograph of the chest in a child having undergone NAI shows multiple posterior rib fractures associated with callus formation (arrows)

are grossly horizontal, parallel to the growth plate, and detach a "frisbee-shaped" bone fragment (i.e., a fragment that is thin centrally and thick peripherally) [31]. On radiographs, metaphyseal fractures appear as "corner" (Fig. 22) or "bucket-handle" (Fig. 23) fractures, depending on the size of the avulsed piece of bone (Fig. 24) and its relation to the X-ray beam. These fractures may be bilateral and symmetric on radiographs, which is another interesting finding in favour of the diagnosis of NAI (Fig. 22).

Complex skull fractures Skull fractures are relatively common in both non-accidental and accidental injuries, and result from direct trauma. Bilateral fractures, fractures involving more than one cranial bone, fractures evidencing a complex configuration (Fig. 25), fractures that cross

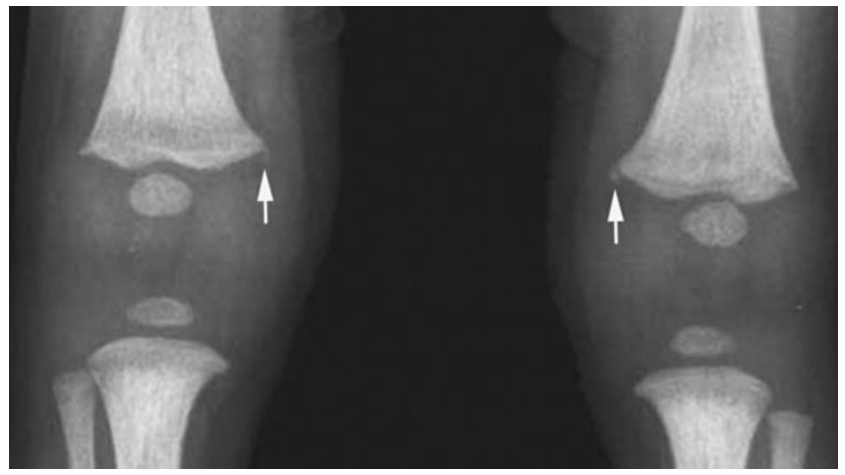

Fig. 22 Anteroposterior radiograph of the knees in a child having undergone NAI reveals a bilateral "corner" fracture (arrows) on the medial aspect of the distal femur

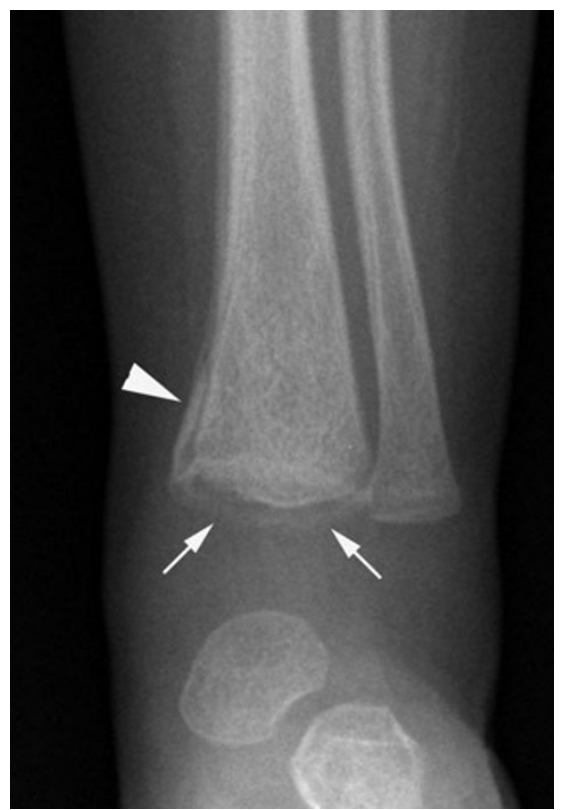

Fig. 23 Anteroposterior radiograph of the ankle in a child having undergone NAI evidences a "bucket-handle" fracture (arrows) of the distal tibia. Since the lesion is extensive, an associated periosteal reaction is seen (arrowhead)

suture lines, depressed, wide (diastasis $\geq 3 \mathrm{~mm}$ ) and growing fractures and fractures associated with underlying intracranial lesions all suggest NAI [31].

Other fractures such as sternal, scapular and spinous process fractures are also highly specific for NAI, but they are rarely encountered in practice.

Other disorders such as copper deficiency, Menkes disease (a genetic disorder of copper metabolism, also known as kinky hair disease), scurvy and vitamin D deficiency rickets may also cause diagnostic difficulties in children with unexplained fractures and be confused in some cases with NAI [33, 34]. However, medical history, clinical manifestations, biochemical and radiological findings allow a correct diagnosis in most cases. In copper deficiency and Menkes disease, fractures are usually noted within the first 6 months of life; they are associated with hypopigmented, and brittle hair and progressive neurologic deterioration in Menkes disease. Typical radiographic findings include osteopenia, bone age retardation, increased density of the provisional zone of calcification and symmetrically distributed metaphyseal abnormalities (cupping and fraying, "sickle-shaped" spurs and fractures) [34]. In scurvy, there is no cupping or fraying of the metaphyses, but radiography shows evidence of radiolucent metaphyseal bands beneath the increased density of the provisional zone of calcification and/or periosteal reaction [34]. In rickets, metaphyseal abnormalities similar to copper deficiency 
Fig. 24 Diagram of a classic metaphyseal lesion depicts a "corner" incomplete fracture (left) and a "bucket-handle" complete fracture (right). $\mathrm{M}=$ metaphysis; $\mathrm{E}=$ osseous portion of the epiphysis; $\mathrm{ca}=$ cartilaginous portion of the epiphysis. The black line corresponds to the fracture line

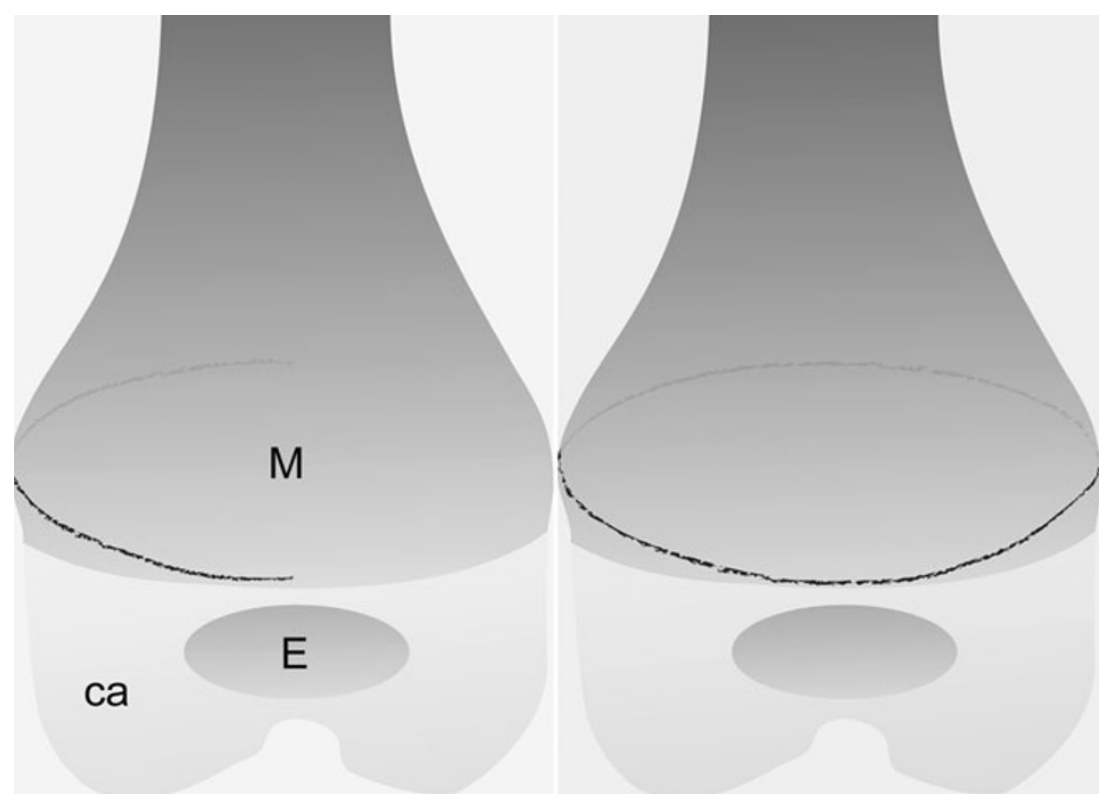

are seen on radiographs but with decreased density of the provisional zone of calcification and increased width of the growth plate [34].

\section{Conclusion}

OI should be suspected in all children presenting with increased bone fragility, including fractures that occur with little or no trauma. Positive diagnosis is typically made on the basis of personal and familial medical history, physical examination (preferably with a clinician who is familiar with OI), radiography and, in some cases, complementary investigations such as bone densitometry, biochemical tests or DNA-based sequencing. On radiographs, OI has no pathognomonic features, but some of them may be suggestive of the diagnosis. Recent advances in the treatment of OI with biphosphonates have resulted in radiographic findings, of which the physician must be aware. Usually differentiating OI from NAI is not difficult in young children, though the issue is often raised in court in the defence of cases of NAI, but if necessary radiography is very useful for the differential diagnosis.
Fig. 25 Anteroposterior and lateral radiographs of the skull in a child having undergone NAI exhibit a complex fracture. The fracture lines (arrows) are bilateral and cross the sutures
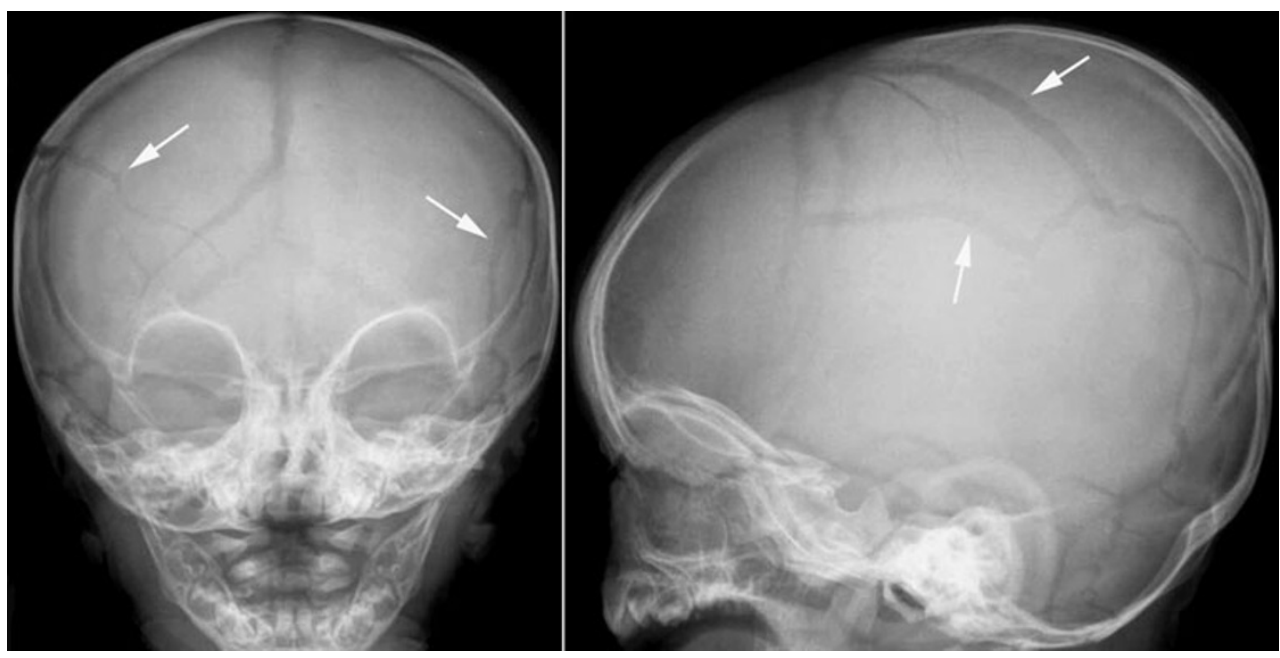
Conflict of interest The authors declare no conflicts of interest. No funding was received for this work.

Open Access This article is distributed under the terms of the Creative Commons Attribution License which permits any use, distribution, and reproduction in any medium, provided the original author(s) and the source are credited.

\section{References}

1. Brusin JH (2008) Osteogenesis imperfecta. Radiol Technol 79:535-548

2. Rauch F, Glorieux FH (2004) Osteogenesis imperfecta. Lancet 363:1377-1385

3. Sillence DO, Senn A, Danks DM (1979) Genetic heterogeneity in osteogenesis imperfecta. J Med Genet 16:101-116

4. Glorieux FH (2008) Osteogenesis imperfecta. Best Pract Res Clin Rheumatol 22:85-100

5. Bulas DI, Stern HJ, Rosenbaum KN, Fonda JA, Glass RB, Tift C (1994) Variable prenatal appearance of osteogenesis imperfecta. J Ultrasound Med 13:419-427

6. Redon JY, Gloaquen D, Collet M, Parent P, Le Grevellec JY (1993) Osteogenesis imperfecta. Reflections after the prenatal diagnosis of 2 cases. J Gynecol Obstet Biol Reprod 22:173-178

7. Solopova A, Wisser J, Huisman TA (2008) Osteogenesis imperfecta type II: fetal magnetic resonance imaging findings. Fetal Diagn Ther 24:361-367

8. Vetter U, Maierhofer B, Müller M, Lang D, Teller WM, Brenner R, Frohneberg D, Wörsdörfer O (1989) Osteogenesis imperfecta in childhood: cardiac and renal manifestations. Eur J Pediatr 149:184-187

9. Charnas LR, Marini JC (1993) Communicating hydrocephalus, basilar invagination, and other neurologic features in osteogenesis imperfecta. Neurology 43:2603-2608

10. Janus GJ, Engelbert RH, Beek E, Gooskens RH, Pruijs JE (2003) Osteogenesis imperfecta in childhood: MR imaging of basilar impression. Eur J Radiol 47:19-24

11. Bachrach LK (2000) Dual energy X-ray absorptiometry (DEXA) measurements of bone density and body composition: promise and pitfalls. J Pediatr Endocrinol Metab 13:983-988

12. Moore MS, Minch CM, Kruse RW et al (1998) The role of dual energy $\mathrm{x}$-ray absorptiometry in aiding the diagnosis of pediatric osteogenesis imperfecta. Am J Orthop 27:797-801

13. Hatz D, Esposito PW, Schroeder B, Burke B, Lutz R, Hasley BP (2011) The incidence of spondylolysis and spondylolisthesis in children with osteogenesis imperfecta. J Pediatr Orthop 31:655-660

14. Ivo R, Fuerderer S, Eysel P (2007) Spondylolisthesis caused by extreme pedicle elongation in osteogenesis imperfecta. Eur Spine J 10:1636-1640

15. Zionts LE, Moon CN (2002) Olecranon apophysis fractures in children with osteogenesis imperfecta revisited. J Pediatr Orthop 22:745-750
16. Tamborlane JW, Lin DY, Denton JR (2004) Osteogenesis imperfecta presenting as simultaneous bilateral tibial tubercle avulsion fractures in a child: a case report. J Pediatr Orthop 24:620-622

17. Semler O, Cheung MS, Glorieux GH, Rauch F (2010) Wormian bones in osteogenesis imperfecta: correlation to clinical findings and genotype. Am J Med Genet A 152A:1681-1687

18. Rieker O, Kreitner KF, Karbowski A (1998) Hyperplastic callus formation in osteogenesis imperfecta: CT and MRI findings. Eur Radiol 8:1137-1139

19. Dobrocky I, Seidl G, Grill F (1999) MRI and CT features of hyperplastic callus in osteogenesis imperfecta tarda. Eur Radiol 9:665-668

20. Cheung MS, Azouz EM, Glorieux FH, Rauch F (2008) Hyperplastic callus formation in osteogenesis imperfecta type V: followup of three generations over ten years. Skeletal Radiol 37:465-467

21. Hui PK, Tung JY, Lam WW, Chau MT (2011) Osteogenesis imperfecta type V. Skeletal Radiol 40:1633

22. Obafemi AA, Bulas DI, Troendle J, Marini JC (2008) Popcorn calcification in osteogenesis imperfecta: incidence, progression, and molecular correlation. Am J Med Genet A 146A:2725-2732

23. Snoeckx A, Vanhoenacker FM, Parizel PM (2008) Popcorn calcifications in osteogenesis imperfecta. JBR-BTR 91:176

24. Glorieux FH (2007) Experience with biphosphonates in osteogenesis imperfecta. Pediatrics 119:S163-165

25. Martinez-Soto T, Pacaud D, Stephure D, Trussell R, Huang C (2011) Treatment of symptomatic osteoporosis in children: a comparison of two pamidronate dosage regimens. J Pediatr Endoncrinol Metab 24:271-274

26. Ward LM, Rauch F, Whyte MP et al (2011) Alendronate for the treatment of pediatric osteogenesis imperfecta: a randomized placebo-controlled study. J Clin Endocrinol Metab 96:355-364

27. Salehpour S, Tavakkoli S (2010) Cyclic pamidronate therapy in children with osteogenesis imperfecta. J Pediatr Endocrinol Metab 23:73-80

28. Panigrahi I, Das RR, Sharda S, Marwaha RK, Khandelwal N (2010) Response to zolendronic acid in children with type III osteogenesis imperfecta. J Bone Miner Metab 28:451-455

29. Grissom LE, Harcke HT (2003) Radiographic features of biphosphonates therapy in pediatric patients. Pediatr Radiol 33:226-229

30. Offiah A, van Rijn RR, Perez-Rossello JM, Kleinman PK (2009) Skeletal imaging in child abuse (non-accidental injury). Pediatr Radiol 39:461-470

31. Lonergan GJ, Baker AM, Morey MK, Boos SC (2003) From the archives of the AFIP. Child abuse: radiologic-pathologic correlation. Radiographics 23:811-845

32. Kleinman PK, Marks SC, Blackbourne B (1986) The metaphyseal lesion in abused infants: a radiologic-histopathologic study. AJR 146:895-905

33. Paterson CR (1990) Osteogenesis imperfecta and other bone disorders in the differential diagnosis of unexplained fractures. J R Soc Med 83:72-74

34. Grünebaum M, Horodniceanu C, Steinherz R (1980) The radiographic manifestations of bone changes in copper deficiency. Pediatr Radiol 9:101-104 